\title{
基于雾线暗原色先验的红外图像去雾算法
}

\author{
左健宏 ${ }^{1}$, 萄素珍 ${ }^{1}$, 禄晓飞 ${ }^{2}$, 李大威 ${ }^{1}$, 李 毅 ${ }^{2}$ \\ (1. 中北大学 大数据学院, 山西 太原 $030051 ; 2$. 酒泉卫星发射中心, 甘肃 酒泉 735000)
}

\begin{abstract}
摘要: 红外图像去雾算法的主要任务是解决红外图像因米氏散射引起的低可见性和模糊。但是当前红 外图像去雾算法对红外图像暗处透射率估计欠佳, 针对这一情况, 研究了基于雾线暗原色先验的红外 图像去雾算法。首先, 利用霍夫变换估计大气光照; 然后, 针对雾线去雾方法在部分场景中失效的现 象, 采用雾线暗原色先验方法, 通过假设雾线较暗端为真实颜色估计透射率, 获取透射率图; 最后为 去除透射率图中噪声, 对透射率图全变分正则化进一步优化透射率图。以公开红外数据库 LTIR 作为 测试对象, 实验结果表明, 本文去雾算法在增强红外图像清晰度的同时未破坏红外辐射分布, 对各种 场景的红外图像有较好去雾效果。透射率估计准确, 有较好红外图像去雾能力。
\end{abstract}

关键词: 红外图像增强; 图像处理; 图像去雾; 全变分; 图像增强

中图分类号：TP751 文献标识码：A 文章编号：1001-8891(2020)06-0552-07

\section{Use of Dark Primary Color Priors for Haze-line-Based Infrared Image Dehazing}

\author{
ZUO Jianhong ${ }^{1}$, LIN Suzhen ${ }^{1}$, LU Xiaofei ${ }^{2}$, LI Dawei ${ }^{1}$, LI Yi ${ }^{2}$ \\ (1. School of Big Data, North University of China, Taiyuan 030051, China; \\ 2. Jiuquan Satellite Launch Center, Jiuquan 735000, China)
}

\begin{abstract}
The main task of infrared image dehazing algorithms is to solve the problems of low visibility and blurring in infrared images; these problems arise from Mie scattering. However, current infrared image dehazing algorithms poorly estimate the dark transmittance of infrared images. Hence, in this study, an infrared image dehazing algorithm is developed based on the dark primary color prior of the haze-line. First, the Hough transform was employed to estimate the atmospheric illumination. Second, a dark primary color prior was employed to address the failure of the haze-line dehazing method in some scenarios. The transmittance was estimated by assuming that the dark end of the haze-line corresponds to the real color, and a transmittance map was obtained. To remove noise in the transmittance map, total variation regularization was used; thus, the transmittance map was optimized. The experimental results obtained using LTIR, a public infrared dataset, as the test dataset show that the proposed algorithm can enhance the clarity of infrared images without affecting the distribution of infrared radiation; in addition, the results show that the proposed algorithm enhances infrared images corresponding to various scenes. The proposed method accurately estimates transmittance and effectively dehazes infrared images.
\end{abstract}

Key words: infrared image enhancement, image processing, image dehazing, total variation, image enhancement

\section{0 引言}

红外图像反映的场景温度分布信息, 对图像融
合、目标检测和视频监控任务有着重要作用 ${ }^{[1-3]}$ 。尽管 红外成像具有全天候的优势, 但在雾䨪天气下, 米氏 散射会削减红外图像的对比度和可见性, 像质退化不

收稿日期: 2019-11-27; 修订日期: 2020-01-20.

作者简介: 左健宏 (1995-), 男, 硕士研究生, 主要从事图像处理, 红外目标检测方面的研究。E-mail: gaoda00123@163.com。

通信作者: 萄素珍 (1966-) , 女, 教授, 博士, 硕士生导师, 主要从事影像大数据处理, 信息融合, 文物虚拟修复方面的研究。E-mail: lsz@nuc.edu.cn。 基金项目：山西省应用基础研究项目（201701D121062），中北大学第十五届科技立项资助项目（2018514）。 
可避免。因此, 研究红外图像去雾算法不但需要增强 其对比度和细节, 同时不能破坏红外图像像素分布信 息，避免出现过增强现象 ${ }^{[4-6]}$

单帧红外图像去雾方法可分为基于红外图像增 强去雾和基于大气散射模型去雾两类。直方图均衡化 是基于红外图像增强去雾的典型代表 ${ }^{[7]}$ 。该方法通过 修正和合并像素值等变换使原图像灰度直方图近似 均匀分布, 增强过程中细节信息丢失, 区域亮度不均 匀。近年来出现的其他基于红外图像增强的去雾方法 还有变分图像增强算法、Retinex 图像增强算法等 ${ }^{[8]}$ 。 这些算法不可避免地会破坏场景原有的温度分布信 息, 对后续的目标检测和场景理解等任务带来不良影 响。基于大气散射模型的去雾算法建立在大气物理模 型基础之上, 保留温度分布信息。此类方法逐渐成为 研究热点。Zheng ${ }^{[9]}$ 借鉴 $\mathrm{He}^{[10]}$ 提出的暗通道先验和暗 通道去雾算法, 同时依托红外图像成像原理, 提出红 外交通图像去雾算法, 加入伽马校正达到了较好的红 外图像去雾效果, 但由于深空等区域普遍温度低, 未被散射而图像中低灰度值像素大量存在, 利用这 类暗区域估计出的透射率不准确是这一类方法的共 性问题, 去雾结果存在严重的过增强和光晕现象。 董浩伟 ${ }^{[11]}$ 对暗通道去雾结果使用 Retinex 算法进行对 比度增强, 提高了去雾结果的对比度和信噪比, 但未 完全解决暗区域透射率估计不准确的问题。

为提升红外图像暗区域去雾效果, 针对大气散射 模型下暗区域透射率估计不佳的问题, 本文以室外场 景红外图像为研究对象, 提出基于雾线暗原色先验的 红外图像去雾算法。首先, 本文通过霍夫变换, 以球 参数空间中点是否落在候选区域内和候选大气光照 是否大于当前像素值为投票条件, 投票选出最优大气 光照; 然后, 将有雾红外图像转换至球参数空间得到 雾线, 依据本文提出的暗原色先验初始化透射率图; 再采用全变分方法优化透射率图, 抑制噪声干扰, 减 少误差; 最后, 通过求解无雾图像实现去雾效果。

\section{1 基于暗原色先验的红外图像去雾算法}

\section{1 大气散射模型}

Narasimhan 提出的大气散射模型（如图 1) ${ }^{[12]}$ 认 为光进入大气层时, 相机接收到的辐射能量实际来源 于两部分: (1)场景受太阳光反射或场景本身辐射的能 量, 这部分能量因场景深度, 大气干扰而有所衰减。

(2)光被散射后形成的大气光。根据此模型, 图像可以 表达为:

$$
I(\chi)=J(\chi) t(\chi)+A(1-t(\chi))
$$

式中: $\chi$ 对应着像素位置 $\chi=(x, y) ; J(\chi)$ 为衰减前图像; $t(\chi)$ 为透射率; $A$ 为全局大气光照; $I(\chi)$ 为雾皬图像。

假设地表大气是均质的 ${ }^{[13]}$, 透射率可表示为:

$$
t(\chi)=\exp [-\beta(\lambda) \mathrm{d}(\chi)]
$$

式中: $d(\chi)$ 代表场景深度; $\lambda$ 代表波长; $\beta(\lambda)$ 为大气散 射系数。

单帧图像去雾问题中, 大气散射模型中 $I(\chi)$ 作为 已知量, 同时求解 $J(\chi) 、 t(\chi) 、 A$ 三个未知量是一个欠 适定问题。需利用各种先验知识从雾霧图像 $I(\chi)$ 估计 大气光照 $A$ 和透射率 $t(\chi)$, 才能求解无雾图像 $J(\chi)$, 如公式(3)所示:

$$
J(\chi)=\frac{I(\chi)-A}{t(\chi)}+A
$$

在热红外成像的地-气辐射传输中, 地面与大气吸 收太阳能, 成为热红外辐射的辐射源, 辐射能量又被 大气多次吸收和散射。因此, 受到大气干扰的红外图 像与可见光雾图呈现出相似的视觉效果。在红外图像 中, 假设地表和大气为朗伯体, 红外辐射衰减与大气 二氧化碳水蒸气吸收、大气中微粒的散射和雨雪影响 相关。文献[11]中红外波段大气辐射衰减为:

$$
t(\lambda)=\exp \left(\begin{array}{l}
\left(-\left(\mu_{\mathrm{H}_{2} \mathrm{O}}+\mu_{\mathrm{CO}_{2}}\right)+\mu_{\mathrm{p}}(\lambda)-\left(\mu_{\mathrm{s}}+\mu_{\mathrm{r}}\right)\right) \\
\cdot d_{\text {infrared }}(\chi)
\end{array}\right)
$$

式中: $\mu_{\mathrm{H}_{2} \mathrm{O}} 、 \mu_{\mathrm{CO}_{2}}$ 为水蒸汽和二氧化碳吸收引起的衰 减系数; $\mu_{\mathrm{p}}(\lambda)$ 为大气微粒的散射衰减系数; $\mu_{\mathrm{s}}$ 为雪引 起的衰减系数; $\mu_{\mathrm{r}}$ 为雨引起的衰减系数; $d_{\text {infrared }}(\chi)$ 为 场景到红外探测器的有效传输距离; $\lambda$ 为波长。

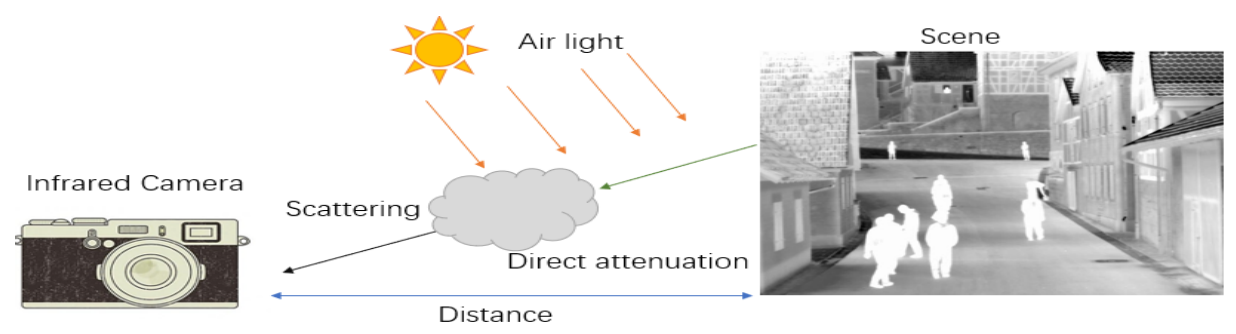

图 1 大气散射模型

Fig. 1 Atmospheric scattering model 
对比式(4)与式(2)可以看出, 红外成像和可见光成 像在雾皬场景下大气透过率估计方法相近, 可以认为 大气散射模型近似适用于红外图像去雾。

\section{2 雾线}

Berman 提出非局部图像去雾方法 ${ }^{[14]}$, 假设无雾 图像可以由远少于颜色空间数的颜色数来近似表达。 从 RGB 空间来看, 一幅图像中像素会聚集成数百个 簇。在雾䨝图像中, 像素建模为原始像素值与大气光 照的凸组合, 原先紧致的簇以直线的形式分布在 RGB 空间中, Berman 将这些线称为雾线, 认为雾线中远 离圆心一端代表原始像素值。

红外图像服从颜色可压缩假设, 可以用较少的颜 色近似表示红外图像, 满足 Berman 非局部去雾方法 假设。因此可将非局部图像去雾方法应用于红外图像 去雾。如图 2 所示, (a)为原图, 图 2(b) (f) 分别采用 聚类数为 $8 、 16 、 32 、 64 、 128$ 的 $k$ 均值聚类对红外 图像进行颜色量化。颜色量化后红外图像可以近似表 示原图像。聚类数为 8、16 颜色量化结果图 2(b), 图 2(c)与图 2(d) (f)（聚类数为 32、64、128 颜色的量 化结果) 相比, 视觉信息损失较大, 可以明显看出阴 影和墙面的不协调。图 2(g)为对 7 个红外场景 100 张 红外图像颜色量化后 PSNR 统计图。

为加快聚类速度, 减少运行时间, 本文采用聚类 数为 32 的 $k$ 均值聚类对红外图像提取雾线。

为获取雾线, 首先将单通道的红外图像转换至 RGB 颜色空间中, 红色分量, 绿色分量, 蓝色分量相 同, 图像视觉信息不发生改变。

$$
I(\chi)=\left[I_{\text {infrared }}(\chi), I_{\text {infrared }}(\chi), I_{\text {infrared }}(\chi)\right]
$$

式中: $I_{\text {infrared }}(\chi)$ 为单通道红外图像, 将单通道红外图 像复制到红色分量, 绿色分量, 蓝色分量中; $I(\chi)$ 为

$$
\underset{A}{\operatorname{argmax}} \sum_{\chi} \sum_{\mathrm{K}} f(\|I(\chi)-A\|) \cdot \Pi[\mathrm{d}(I(\chi),(A, \varphi, \theta))<\imath] \cdot \Pi[A>I(\chi)]
$$
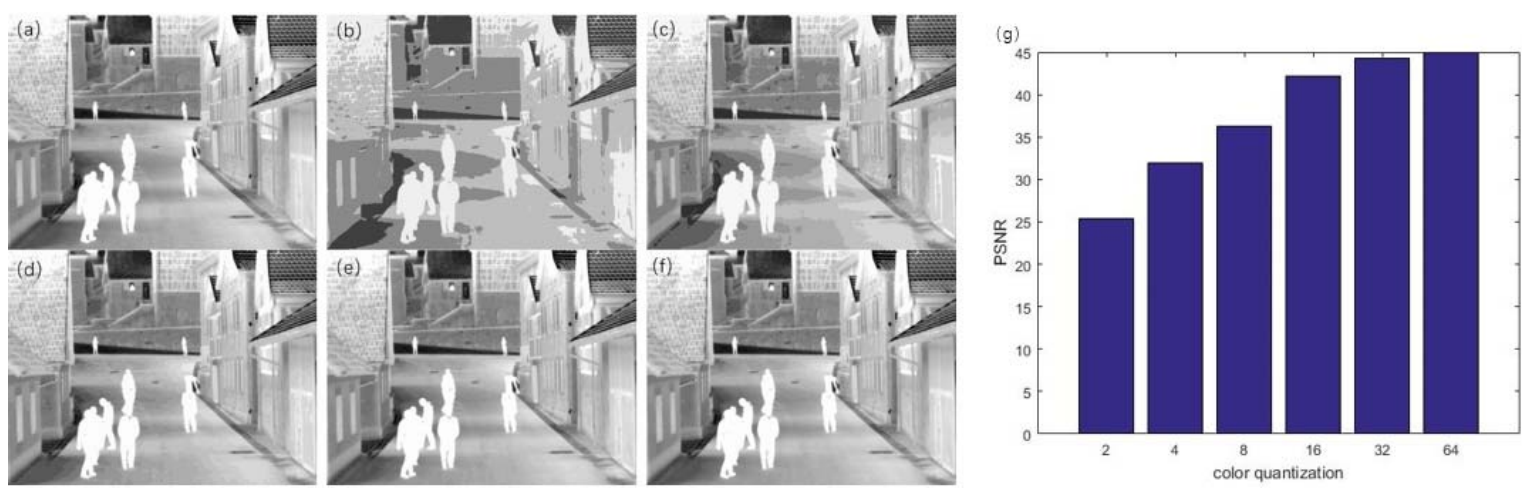

(a) 红外图像 (b) $n=8$, 颜色量化 (c) $n=16$, 颜色量化 (d) $n=32$, 颜色量化 (e) $n=64$, 颜色量化 (f) $n=128$, 颜色量化 (g) PSNR
(a) Infrared image
(b) $n=8$ color quantization
(c) $n=16$ color quantization
(d) $n=32$ color quantization
(e) $n=64$ color quantization

(f) $n=128$ color quantization (g) PSNR

图 2 红外图像颜色可压缩验证 Fig. 2 Verify the compressibility of infrared image color 


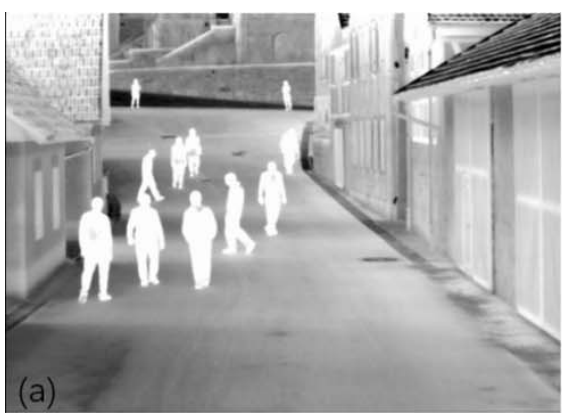

(a) 红外图像

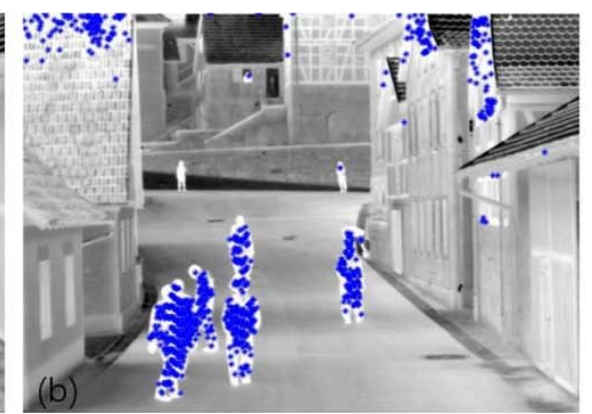

(b) 红外图像中聚类标记

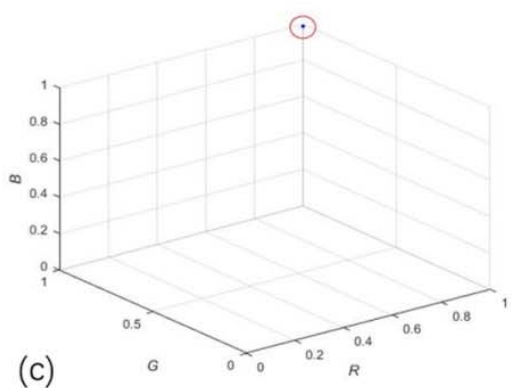

(c) RGB 颜色空间中的标记像素

(a) Infrared image

(b) Clustering marker pixel in infrared image

(c) Marked pixels in RGB color space

图 3 红外图像雾线

式中: $f(\|I(\chi)-A\|)$ 为衰减系数, 能够加速迭代过程。

$\Pi[\bullet]$ 为指示函数, 内部不等式成立则指示函数值为 1 , 反之为 0 。 $i$ 为阈值, $\mathrm{d}[I(\chi),(A, \varphi, \theta)]<l$ 作用为判断当前 候选大气光照下, 将图像变换到球参数空间后, 像素 点是否落阈值范围内。 $\Pi[A>I(\chi)]$ 判断当期候选大气 光照是否大于当前像素值。

\section{4 雾线暗原色先验下的透射率估计}

由式(9)可知, 径向距离 $r(\chi)$ 的不同意味着透射率 的不同。对于一条给定的雾线, 径向距离 $r(\chi)$ 由无雾 图像 $J(\chi)$, 大气光照 $A$ 和透射率 $t(\chi)$ 共同决定:

$$
r(\chi)=t(\chi)\|J(\chi)-A\| 0 \leq t(\chi) \leq 1
$$

研究中发现, 对于红外图像中一条给定的雾线, 亮度更高的像素受散射影响变暗落入此簇, 因此提出 用雾线暗原色描述红外图像中的原始大量的暗区域, 同时不会对亮区域造成影响。

对透射率 $t(\chi)$ 取最小值:

$$
r_{\min }=t_{\min }(\chi)\|J(\chi)-A\|
$$

式中: $r_{\min }$ 代表此雾线最小径向距离; $t_{\min }(\chi)$ 代表最小 透射率。联系公式(13)、(14), 得出透射率:

$$
t(\chi)=\frac{r(\chi) t_{\min }(\chi)}{r_{\min }}
$$

此时, 求透射率问题转换为估计雾线最小透射率 问题。根据暗通道先验, 由公式(1)可知, 对于每条雾
Fig. 3 Infrared image haze lines

线, 最小透射率 $t_{\min }(\chi)$ 可表示为:

$$
t_{\min }(\chi)=\min \left\{1-\min \left\{\frac{I_{\text {line }}(\chi)}{A}\right\}\right\}
$$

式中: $I_{\text {line }}(\chi)$ 为雾线内元素在有雾红外图像中的像素 值, 通过最小化暗通道先验的透射率估计, 计算每条 雾线的最小透射率。

将式(16)代入式(15), 透射率可进一步写为:

$$
t(\chi)=\frac{r(\chi) \cdot\left(\min \left\{1-\min \left\{\frac{I_{\text {line }}(\chi)}{A}\right\}\right\}\right)}{r_{\min }}
$$

\section{5 透射率优化}

为了取得更好的去雾结果, 使用全变分正则优化 透射率图, 对求得的透射率进行保边平滑, 抑制噪声 干扰, 减少误差:

$$
\min \left\|t(\chi)-t_{\text {refine }}(\chi)\right\|^{2}+\lambda_{\mathrm{tv}}\|\nabla t(\chi)\|_{1}
$$

式中: $\lambda_{\mathrm{tv}}$ 为正则项参数; $\nabla t(\chi)$ 为梯度算子, $t_{\mathrm{refine}}(\chi)$ 优化后透射率, 迭代求解公式(16), 得到优化后的透 射率 $t_{\mathrm{refine}}(\chi)$ 。 $\lambda_{\mathrm{vv}}$ 调整图像平滑程度, $\lambda_{\mathrm{tv}}$ 越大, 图像 平滑程度越高, 降噪效果好, 但破坏图像结构信息; 反之, $\lambda_{\mathrm{tv}}$ 值越小, 降噪效果不明显, 无法优化透射率 图, 不同 $\lambda_{\mathrm{tv}}$ 优化结果如图 4 所示。

将 $t_{\text {refine }}(\chi) 、 A$ 代入公式(3)中求得去雾图像 $J(\chi)$, 将去雾结果转为灰度图即为最终去雾结果 (如图 5)。

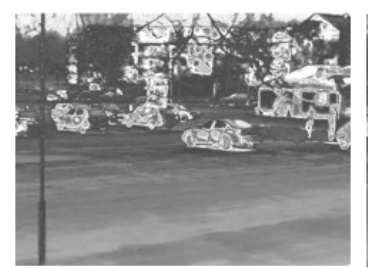

(a) Original image

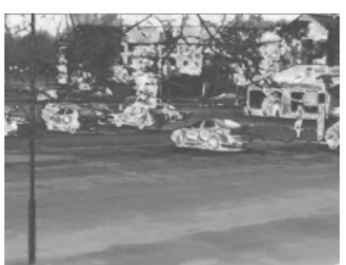

(b) $\lambda=0.005$

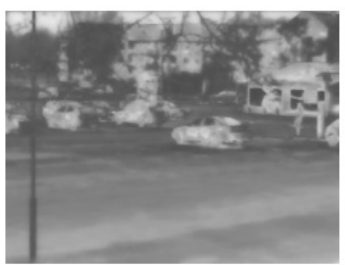

(c) $\lambda=0.01$

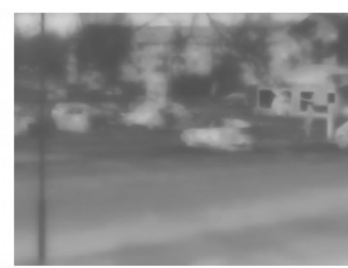

(d) $\lambda=0.05$

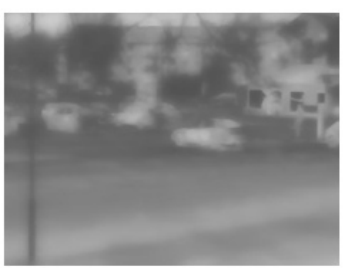

(e) $\lambda=0.07$

图 4 透射率优化

Fig.4 Transmittance optimization 


\section{2 分析与讨论}

为了验证本文提出算法的有效性, 选取林雪平大 学热红外数据库 ${ }^{[16]}$ (Linkoping Thermal InfraRed, LTIR）中 3 个不同场景的图像进行分析, 包含远景, 近景, 浓雾, 昏暗等情况, “行人”, “挖掘机”, “公 园” 分别对应图 6 中场景 $1 \sim$ 场景 3 。

在所有实验中, 依据本文 1.2 节所述, 在聚类数 为 32 时, 红外图像颜色量化效果较好, 能有效降低 聚类所消耗的时间, 因此本文算法的球面空间采样数 $K=32$ 。式(12)中阈值参数作用限制采样区域, 依据经 验设置 $l=0.02$ 。在透射率优化部分, 式(18)中正则项 参数过小时降噪效果不佳, 过大时透射率图过度模 糊, 经过实验后设置 $\lambda_{\mathrm{tv}}=0.01$ 。

本文算法与直方图均衡化图像增强算法 ${ }^{[7]}$, 红外 交通图像去雾算法 ${ }^{[9]}$ 、大气散射模型和 Retinex 增强 去雾算法 ${ }^{[11]}$ 和 Berman 非局部去雾算法 ${ }^{[14]}$ 进行比较。

\section{1 主观评价分析}

主观分析实验结果, 图 6 各个场景中, (a)为原始 红外图像, (b)为文献[7]提出的直方图均衡化算法, (c) 为文献[9]中 Zheng 提出的红外交通图像去雾算法, (d) 为文献[11]中董浩伟提出的基于大气散射模型和 Retinex 增强的去雾方法, (e)为文献[14]中 Berman 提 出的非局部去雾方法, (f)为本文方法。

对比结果如图 6 所示, 可以看出, 直方图均衡化 算法通过灰度级合并增强图像对比度, 对有雾红外图 像增强有限, 在远景场景 1 和场景 2 中由于行人、挖 掘机中灰度相近, 灰度合并后丢失的细节信息, 同样 的, 场景 3 处理结果中树木纹理模糊, 过增强现象严 重。Zheng 通过对暗通道去雾结果进行伽马校正来优 化去雾结果, 但伽马校正鲁棒性较差, 同一伽马参数 无法适用于不同场景。在场景 1 中伽马校正后有效突 出行人, 但削弱了背景。昏暗场景 2 和 3 中, 图像整 体较暗, 透射率估计错误, 伽马校正后, 挖掘机、行
人过增强严重, 丢失场景细节。董浩伟通过对暗通道 去雾后的红外图像进行 Retinex 增强。一定程度上改 善了图像昏暗的问题, 在场景 1 中有效突出了草地和 灌木从的对比度, 视觉效果较好, 但破坏了红外图像 的辐射分布。在场景 3 中, 由于去雾结果不好, 在 Retinex 增强后放大图像噪声, 视觉效果较差。Berman 基于颜色可压缩假设提出非局部去雾算法, 将图像转 换至球参数空间获取雾线, 利用雾线估计透射率。 Berman 实验结果对比度有所提高, 去雾效果较好, 细节得以突出。由于 Berman 的方法将雾线中亮端假 设为真实颜色, 认为此处透过率为 1 , 导致在近景场 景 1 中树干纹理等较亮处过增强现象明显, 破坏了红 外图像辐射分布。本文方法实验结果中, 由于用雾线 中最小径向距离和最小透射率估计透射率, 在场景 1 草坪区域中, 场景 2 沟壑中, 场景 3 椅子中有更多细 节, 透射率估计准确, 对红外图像中暗区域处理更佳, 保留较多的细节信息, 改善图像的视觉效果。对场景 1 中树木和草地、场景 2 中挖掘机、场景 3 中公园设 施都得到有效增强。但在场景 1 中, 去雾不完全, 可 以看出背景中仍含有一层薄雾未去除。

\section{2 客观评价}

由于难以获取与有雾红外图像相对应的无雾红 外图像, 无法通过对无雾红外图像与去雾结果比较进 行直接评价, 为客观评价实验结果, 从提高图像清晰 度和尽可能保留原始红外图像辐射分布的角度对去 雾结果进行评价。

由香农提出的信息熵 (information entropy, IE) 是 衡量图像清晰度的有效指标, 能有效衡量图像信息丰 富程度, 判断是否较好地保持了原图像细节信息。信 息熵越大，图像所含信息量越大。

结构相似性（structural similarity index, SSIM）通 过对比图像的对比度、亮度和结构信息的相似性来评 价图像质量, 能有效判断结果图像与原图像辐射分布 是否一致。

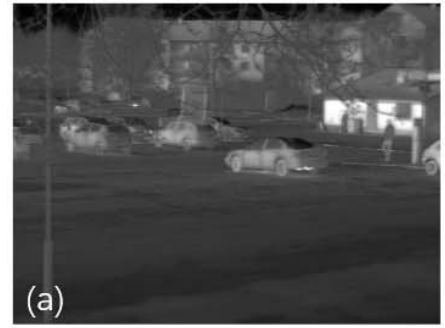

(a) 红外图像

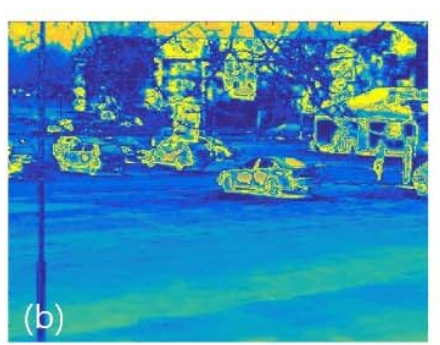

(b) 初始透射率图

(b) Initial transmission 图 5 红外图像去雾

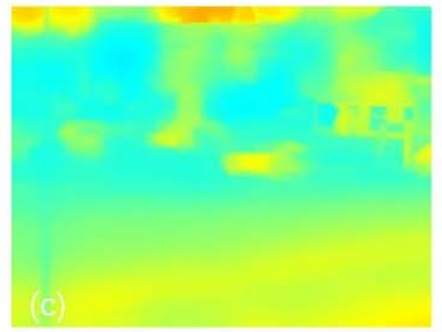

(c) 优化后透射率

(c) Estimation transmission
Fig. 5 Infrared image dehazing

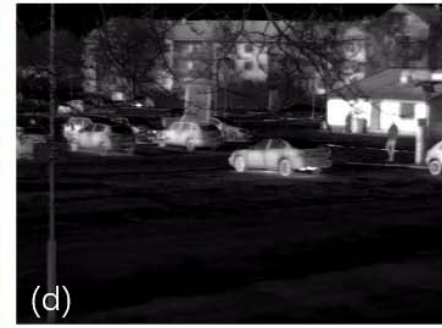

(d) 去雾结果

(d) Dehazing result 

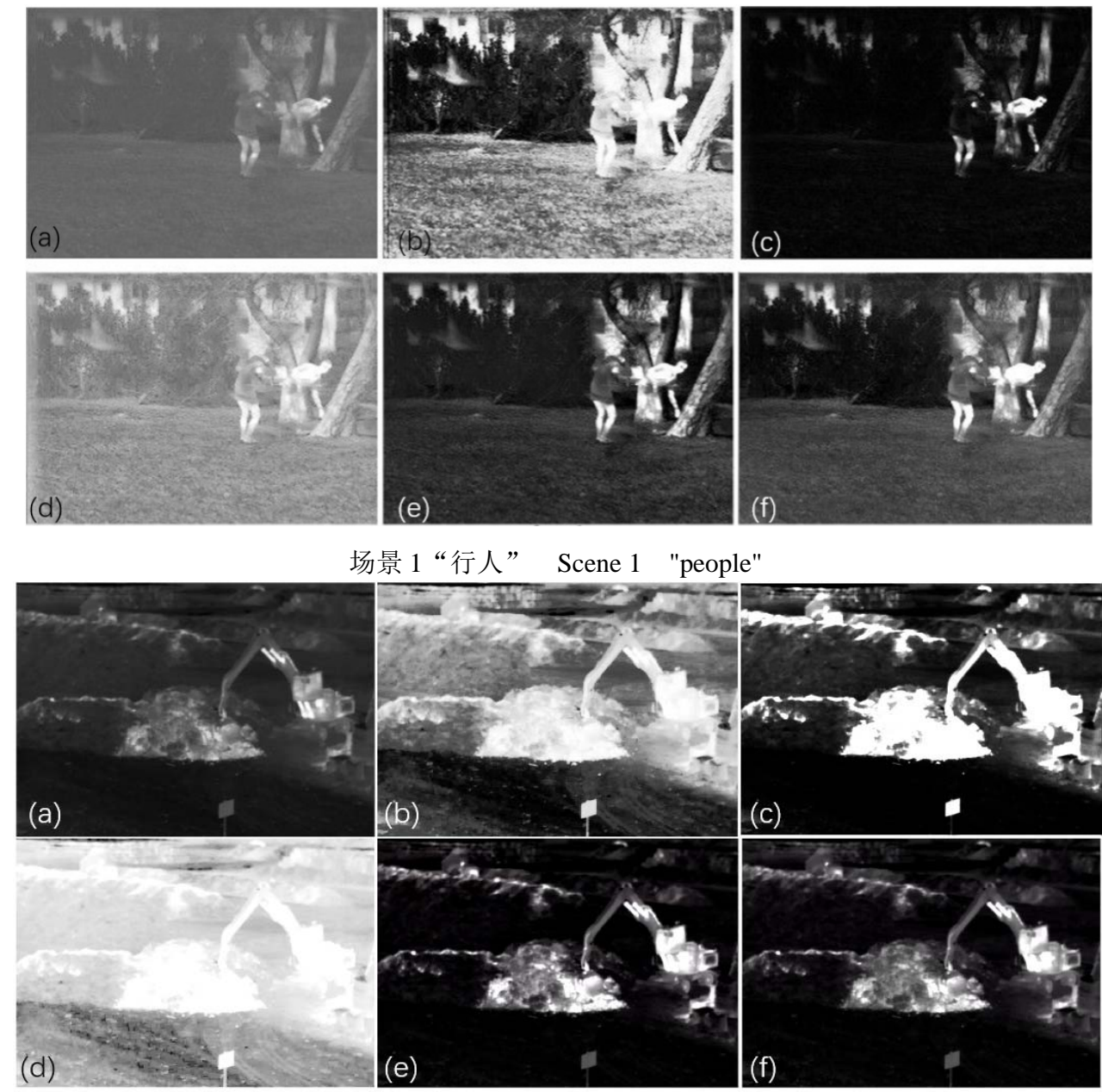

场景 2 “挖掘机” Scene 2 "excavator"
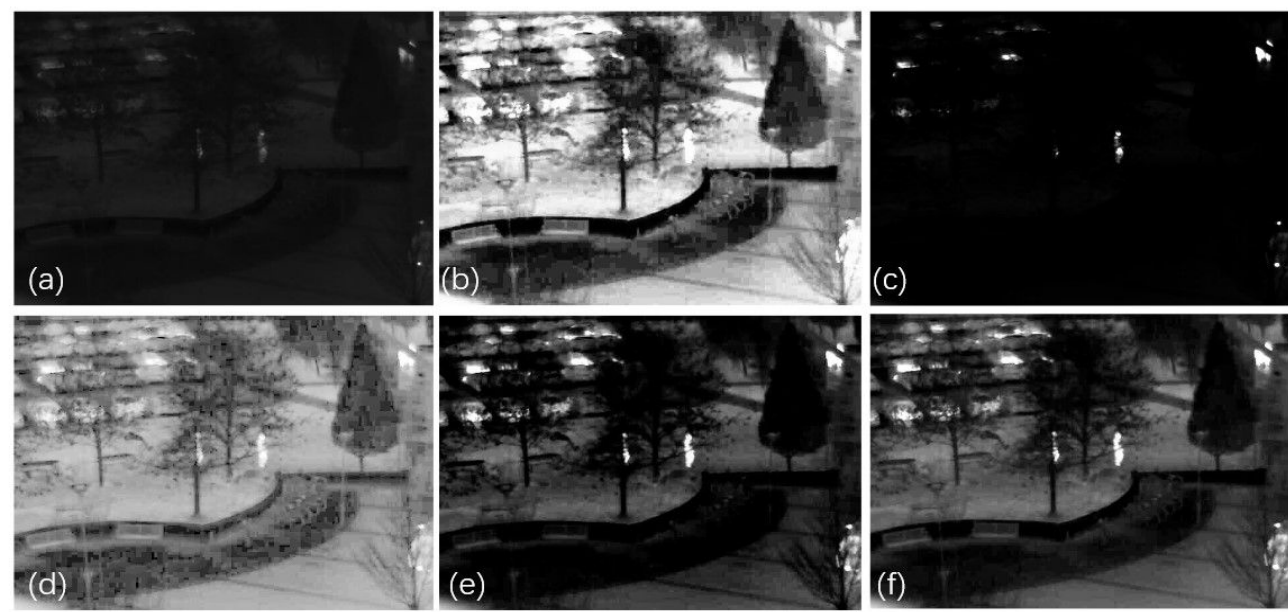

场景 3 “公园” Scene 3 "park "

图 6 不同算法去雾结果，各场景分别为: (a) 原始图像; (b) 文献[7]方法; (c) 文献[9]方法; (d) 文献[11]方法; (e) 文献[14]方法; (f) 本文方法

Fig.6 Different dehazing algorithms results in each scene: (a)Original image; (b) The reference [7]; (c) The reference [9]; (d)The reference [11]; (e)The reference [14]; (f) Our result

因此, 使用结构相似性（structural similarity 示。可以看到本文算法在场景 1 中增强了行人的对 index, SSIM) 作为评价指标, 衡量实验结果的清晰 度和保留原图红外辐射分布的能力, 结果如表 1 所 比度，在场景 2 中，本文去雾不完全导致信息熵较 低, 而文献[7]方法有效增强背景中土坡和沟壑的对 
表 1 红外图像去雾定量评价 Table 1 Quantitative evaluation of infrared image dehazing

\begin{tabular}{ccccccc}
\hline Image & Quality evaluator & {$[7]$} & {$[9]$} & {$[11]$} & {$[14]$} & Ours \\
\hline Scene 1 & IE & 5.2078 & 4.8575 & 5.8149 & 6.5479 & 7.1213 \\
Scene 1 & SSIM & 0.3589 & 0.2169 & 0.7185 & 0.5760 & 0.7357 \\
Scene 2 & IE & 6.2322 & 6.0449 & 6.2118 & 5.6855 & 6.2242 \\
Scene 2 & SSIM & 0.5114 & 0.4794 & 0.3271 & 0.4239 & 0.5572 \\
Scene 3 & IE & 4.6417 & 2.4400 & 5.9449 & 5.8779 & 6.9328 \\
Scene 3 & SSIM & 0.2074 & 0.1293 & 0.1969 & 0.4359 & 0.5500 \\
\hline
\end{tabular}

比度, 但挖掘机过增强现象严重。总体来看, 本文 算法取得了较好的去雾效果, 去雾图像提升清晰度 的同时, 保持了和原图像相似的辐射分布, 不会对 后续工作造成阻碍。总体上, 本文方法效果更佳, 与主观评价结果一致。

\section{3 结论}

本文提出基于暗原色先验的红外图像非局部去 雾算法, 通过霍夫变换估计大气光照, 针对红外图像 含深空场景时透射率估计不佳这一问题, 采用暗原色 先验方法, 利用雾线估计透射率, 提升去雾能力和场 景适应能力。研究了全变分正则化方法优化透射率 图, 来降低噪声对估计透射率的影响。实验结果表明, 暗原色先验能够对红外图像暗区域更好的建模, 使用 本文算法能有效处理较暗红外图像雾霔, 去雾结果细 节纹理清晰, 具有较好视觉效果。但在部分场景下去 雾不完全, 需要进一步改进。

\section{参考文献:}

[1] YU Z, WANG X, BI X, et al. A light dual-task neural network for haze removal[J]. IEEE Signal Processing Letters, 2019, 25(8): 1-1.

[2] 曹慧, 张宝辉, 陈磊, 等. 基于伪暗原色的红外增强技术研究[J]. 红 外技术, 2016, 38(6): 476-480.

CAO H, ZHANG B H, CHEN L, et al. Research of infrared images enhancement technique based on pseudo dark channel prior[J]. Infrared Technology, 2016, 38(6): 476-480.

[3] 杨元庆, 张志利, 侯传勋. 一种近地背景下红外弱小目标检测预处理 算法[J]. 红外技术, 2018, 40(8): 812-817.

YANG Y Q, ZHANG Z L, HOU C X. A preprocessing algorithm for infrared small-target detection in the near-earth background[J]. Infrared Technology, 2018, 40(8): 812-817.

[4] 丁畅, 董丽丽, 许文海. 海面目标的红外景象增强技术研究 [J]. 光学 学报, 2018, 38(6): 0610001.

DING C, DONG L L, XU W H. Enhancement technique for infrared scene with maritime target[J]. ACTA OPATICA SINICA, 2018, 38(6): 0610001.

[5] BAI X. Morphological infrared image enhancement based on multi-scale sequential toggle operator using opening and closing as primitives[J]. Infrared Physics \& Technology, 2015, 68:143-151.

[6] Hassan H, LUO B, XIN Q, et al. Single image dehazing from repeated averaging filters[C]//2019 IEEE 8th Joint International Information Technology and Artificial Intelligence Conference (ITAIC), 2019: 1053-1056.

[7] WANG Q , Ward R K . Fast image/video contrast enhancement based on weighted thresholded histogram equalization[J]. IEEE Transactions on Consumer Electronics, 2007, 53(2): 757-764.

[8] TENG L, XUE F, BAI Q. Remote sensing image enhancement via edge -preserving multiscale retinex[J]. IEEE Photonics Journal, 2019, 11(2): $1-10$.

[9] ZHENG L , SHI H, GU M. Infrared traffic image enhancement algorithm based on dark channel prior and gamma correction[J]. Modern Physics Letters B, 2017, 31(19-21): 84-92.

[10] HE K, SUN J, TANG X. Single image haze removal using dark channel prior[J]. IEEE Transactions on Pattern Analysis \& Machine Intelligence, 2011, 33(12): 2341-2353.

[11] 董浩伟, 陈洁. 一种基于大气散射模型和 Retinex 的红外图像去雾算 法[J]. 红外技术, 2019, 41(4): 347-356.

DONG H W, CHEN H. Infrared image defogging algorithm based on atmospheric scattering model and Retinex[J]. Infrared Technology, 2019, 41(4): 347-356.

[12] Nayar S K, Narasimhan S G. Vision in bad weather[C]//Proceedings of the Seventh IEEE International Conference on Computer Vision, 1999, 2: 820-827.

[13] ZHANG W, LU J, XU X, et al. Estimation of atmospheric light based on Gaussian distribution[J]. Multimedia Tools and Applications, 2019, 78(11): DOI: 10.1007/s11042-019-7401-2.

[14] Berman D, Avidan S. Non-local image dehazing[C]//Proceedings of the IEEE conference on computer vision and pattern recognition, 2016: 1674-1682.

[15] Berman D, Treibitz T, Avidan S. Air-light estimation using haze-lines[C]//2017 IEEE International Conference on Computational Photography (ICCP), 2017: 1-9.

[16] Berg A, Ahlberg J, Felsberg M. A thermal object tracking benchmark[C]//12th IEEE International Conference on Advanced Video and Signal Based Surveillance (AVSS), 2015: 1-6. 